This is the final peer-reviewed accepted manuscript of:

T. Di lanni, L. De Marchi, A. Perelli and A. Marzani, "Compressive sensing of full wave field data for structural health monitoring applications" in IEEE Transactions on Ultrasonics, Ferroelectrics, and Frequency Control, vol. 62, no. 7, pp. 1373-1383, July 2015

The final published version is available online at:

https://doi.org/10.1109/TUFFC.2014.006925

Rights / License:

The terms and conditions for the reuse of this version of the manuscript are specified in the publishing policy. For all terms of use and more information see the publisher's website.

This item was downloaded from IRIS Università di Bologna (https://cris.unibo.it/)

When citing, please refer to the published version. 


\title{
Compressive Sensing of Full Wavefield Data for Structural Health Monitoring Applications
}

\author{
Tommaso Di Ianni, Luca De Marchi, Member, IEEE, Alessandro Perelli, \\ and Alessandro Marzani
}

\begin{abstract}
Numerous non destructive evaluations and structural health monitoring approaches based on guide waves rely on analysis of wavefields recorded through scanning laser Doppler vibrometers (SLDVs) or ultrasonic scanners. The informative content which can be extracted from these inspections is relevant. However, the acquisition process is generally time-consuming posing a limit in the applicability of such approaches. In order to reduce the acquisition time, in this work a random sampling scheme based on Compressive Sensing (CS) is used to minimize the number of points in which the field is measured. The CS reconstruction performance is mostly influenced by the choice of a proper decomposition basis to exploit the sparsity of the acquired signal. Here, different basis have been tested to recover the guided waves wavefield acquired on both an aluminum and a composite plate. Experimental results show that the proposed approach allows to reduce the measurement locations required for accurate signal recovery to less than $34 \%$ of the original sampling grid.
\end{abstract}

\section{Keywords}

Lamb waves, Compressive sensing, Defect detection, NDE, Full wavefield imaging.

\section{INTRODUCTION}

During last decades, the constant need to improve the operational safety of structures is driving the development of nondestructive evaluation methods and monitoring systems aimed at the ongoing assessment of the health conditions of the structures. Structural health monitoring (SHM) includes a wide range of diagnostic techniques that allow the real-time evaluation of both the existence and entity of defects, reducing life-maintenance costs of structural components. For what concern plate-like components, diagnostic methods bases on guided wave [1], [2] have emerged as a viable option among ultrasonic methods because of the ability of such waves to travel long distances with reduced energy loss. 
In particular, the visualization of the guided waves full wavefield propagating into the structure can show the waves interaction with structural features, turning out to be a suitable tool to automatically detect the presence of flaws [3].

A common way to obtain wavefield data consists of exciting the structure using a piezoelectric PZT transducer while acquiring guided wave responses at the user-defined spatial and temporal resolution by means of a scanning laser Doppler vibrometer [4], [5]. Such vibrometer measures the out-of-plane surface velocity in a grid of equispaced points over the structure. In such application, to reduce the effect of acquisition noise, multiple time traces are acquired at every scan point, making such a process rather time consuming.

However, if the wavefield signal can be sparsely decomposed in a limited number of basis functions a novel sensing paradigm can be applied. Such paradigm is based on the Compressive Sensing theory [6] which asserts that, thanks to sparsity, a signal can be acquired and recovered from a limited number (much smaller than what Shannon/Nyquist theorem suggests) of linear measurements without loss of information. CS methods have been used for ultrasonic data reduction [7], [8], [9], [10], and in exploration seismology [11] [12] to speed up the data acquisition phase. In these latter papers, it has been shown that a very good reconstruction of the wavefield can be achieved by using a random sampling procedure with some constraints enforced to limit the maximum sample distance, i.e. spatial gap.

In the contest of guided wave inspections, CS has been used as a powerful mean to perform signal decomposition and analysis [13], to extract the multimodal dispersion curves [14] as well as to expedite the acquisition process in ultrasonic propagation imaging [15]. In this work, a CS-based approach aimed at minimizing the SLDV full wavefield acquisiton phase is presented.

Besides the different application domains, CS methodologies can be distinguished on the basis of two distinctive features: (i) the selected dictionary of basis functions which produces the sparse representation of the considered signal, and (ii) the measurement mechanism, which substitutes the traditional Nyquistbased data collection techniques.

A variety of dictionaries have been developed for representing multidimensional signals [16]. These dictionaries can be either based on an analytic formulation (i.e. Analytical Dictionaries such as Fourier, Wavelets, Gabor atoms), or defined on the basis of a set of realizations of the data (Learned Dictionaries). In this work, Analytical Dictionaries have been used. In fact, the characteristic of guided wave propagation are strongly dependent on the material and geometrical properties of the inspected medium, and this would require a specific and time-consuming dictionary learning procedure applied each time the material or simply the thickness or temperature of the inspected material change. Analytical dictionaries may be less 
effective than Learned Dictionaries for a given experimental setup, but can be fruitfully employed in many applicative contexts without any repeated training. Beside of that, analytical formulations allow for fast implicit decompositions, and consequently less computationally-intensive signal recovery procedures.

In our approach, the number of scan points acquired in a standard laser vibrometer acquisition is decremented by using two different subsampling techniques: the first one is the so called jittered sampling which as been already used in seismic applications [11]; the second one is the Farthest point sampling [17] which is based on an iterative strategy.

The implemented procedure takes into account both suitable sparsity-promoting domains and samplepoints distribution strategies, achieving the best recovering result possible starting from highly incomplete wavefield data. In order to prove its effectiveness, the approach has been validated on signals from experimental setups with different kinds of structural defects.

The paper is organized as follows: Section II deals with the definition of CS theoretical framework. Two approaches for sampling-points distribution are described in Section III, whereas a review of sparsity promoting decomposition bases suited for guided wave inspections is provided in Section IV. In Section $\mathrm{V}$, experimental results concerning the reconstruction of the sub-sampled wavefield are shown. Finally, conclusions and further developments are summarized in Section VI.

\section{Compressive Sensing of WAVEFIEld Data}

Compressive Sensing [18] is a theoretical framework which relies on two principles, namely sparsity and incoherence. The first one expresses the idea that a signal's information content can be represented into a small amount of data when expressed in terms of a proper basis. While incoherence is related to the idea that the elements of the sparsifying basis are poorly correlated with the sampling functions.

Let $\Sigma(x, y, t)$ be the wavefield signal in the spatial and temporal domain, $s \in \mathbb{R}^{n}$ is its sampled version, acquired according the Nyquist sampling theory. We are interested to recover $s$ from a small set of linear measurements

$$
y_{i}=\left\langle\Sigma(x, y, t), \varphi_{i}(x, y)\right\rangle, \quad i=1, \ldots, m
$$

being $\varphi_{i}$ the sampling functions. In under-sampled situations the number of available measurements $m$ is much smaller than the dimension of the signal $n$, and the recovery problem is in general ill-posed and admits infinite solutions. Such limitation can be overcome if some further assumptions can be posed.

Let us consider a vector $\alpha \in \mathbb{R}^{n}$ such that $s=\Psi \alpha$, with $\Psi \in \mathbb{R}^{n \times n}$. We define the representation produced by the basis $\Psi$ as $K$-sparse if $K$ is the number of nonzero elements of $\alpha$. Furthermore, 


$$
m \geq C \cdot \mu^{2}(\boldsymbol{\Phi}, \boldsymbol{\Psi}) \cdot K \log (n)
$$

In practical applications, measurements are affected by noise:

$$
y=\boldsymbol{\Phi} \boldsymbol{\Psi} \alpha+z
$$

where $z$ is the noise term. Moreover, real world data are not exactly sparse. Despite this fact, when the coefficients of vector $\alpha$ decay exponentially in absolute value, the signal is still compressible, and the approximation of $\alpha$ which retains its $\mathrm{K}$ largest entries can be recovered [6] by solving the following sparsity-promoting problem:

$$
\min \|\tilde{\alpha}\|_{\ell_{1}} \quad \text { subject to } \quad\|\boldsymbol{\Phi} \boldsymbol{\Psi} \tilde{\alpha}-y\|_{\ell_{2}} \leq \epsilon,
$$

where $\epsilon$ bounds the amount of noise in the data.

\section{SAMPLING-POINTS DISTRIBUTION PATTERNS}

As detailed in the previous section, the design of an efficient compressive sensing protocol requires to define a suitable sampling scheme $(\boldsymbol{\Phi})$ and the sparsity-promoting dictionary $\boldsymbol{\Psi}$ in order to satisfy the incoherence condition(2). In this work, the waveform measurement is the one produced by scanning devices such as SLDVs in the spatial and temporal domain, and $n$ is the number of samples which should be acquired to respect the Shannon sampling theory (i.e.the full-resolution sampling grid). By assuming that the SLDV measurements are almost ideally point-like, the sub-sampling matrix $\boldsymbol{\Phi}$ is given by the Dirac (identity) basis in which are removed the rows corresponding to $n-m$ locations. In this section, different strategies to select the preserved $m$ samples are presented and discussed. It should be anticipated that the subsampling is performed just in the spatial domain, since no advantage in terms of reconstruction accuracy or reduction of measurement time is achieved by subsampling the wavefield also in the time domain. 


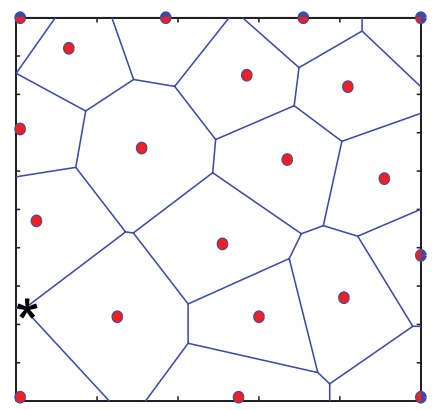

(b)

Fig. 1: (a) Jittered subsampling scheme, the dots represent the scanpoints obtained by regularly subsampling the full resolution grid, their position is then randomly perturbed (bigger circles) to define the scanpoint to be acquired in the CS procedure; (b) Farthest Point sampling, the circles represent the initial set of sampling points; such set is enlarged by selecting iteratively the farthest (marked by an asterisk) among the vertices of the Voronoi diagram (solid lines).

For each step of the FPS procedure, the farthest point is found by computing the Voronoi diagram [22] from the previous samples, and then selecting the farthest among the Voronoi diagram vertices as 
additional sampling point. When the Voronoi diagrams are computed on the basis of the pure Euclidean distance, the FPS method progressively limits the maximum gap between sampling points, thus producing a rather uniform sampling distribution. When needed, the sample density can be also adaptively varied by following the strategy presented in [17].

\section{SPARSITY-PROMOTING DICTIONARIES FOR WAVEFIELD IMAGES}

Let us focus now the discussion on the matrix $\Psi$. Such matrix transforms the signal from the sparse representation domain to the domain in which the measurements are acquired (i.e. the spatial and temporal domain, as specified in the previous section). In this work, we will evaluate as sparse representation domains the ones spanned by the Fourier exponentials, Curvelets, or Wave Atoms. This means that $\Psi$ will be the matrix operator which computes the Fourier, Curvelet or Wave Atom inverse transforms, respectively.

\section{A. Fourier domain}

The dispersive and multi-modal characteristics of guided wave propagation have been extensively studied. A guided wave which travels a distance $\left|x-x_{s 1}\right|$ from an ideal actuator (point-like source) can be modelled in the frequency domain as a dispersive system whose response is:

$$
S_{0}(f, x)=S_{0}\left(f, x_{s 1}\right) \cdot \sum_{i=1}^{M} A_{M}(f) e^{-j\left|x-x_{s 1}\right| k_{M}(f)}
$$

being $S_{0}\left(f, x_{s 1}\right)$ the Fourier Transform of the excitation pulse at the actuation position $x_{s 1}, k_{M}(f)$ the frequency-wavenumber dispersion curve for mode $M$ and $A_{M}(f)$ its dispersive attenuation in the range of frequencies considered. It follows that in the Fourier domain, the full wavefield components are concentrated on the dispersion curves $k_{M}(f)$ of the propagating wavemodes. The sparsifying effect of the Fourier decomposition can be observed in Fig. 2.

Space-time traces related to the simulation of Lamb wave propagation in a $1 \mathrm{~mm}$-thick aluminum plate, considering a 3-cycle sine wave centered at $30 \mathrm{kHz}$ as excitation pulse, are depicted in Fig. 2(a). More specifically, the Semi-analytical Finite Element (SAFE) formulation developed in [23] has been used to compute the time waveforms at different distances, considering the following nominal properties for aluminum: Young modulus $E=69 \mathrm{GPa}$, Poisson's coefficient $\nu=0.33$ and density $\rho=2700$ $\mathrm{kg} / \mathrm{m}^{3}$. The frequency/wavenumber representation of the same signal is depicted in Fig. 2(b). It is worth noticing that the Fourier representation of the simulated wavefield is much sparser than the time-space 

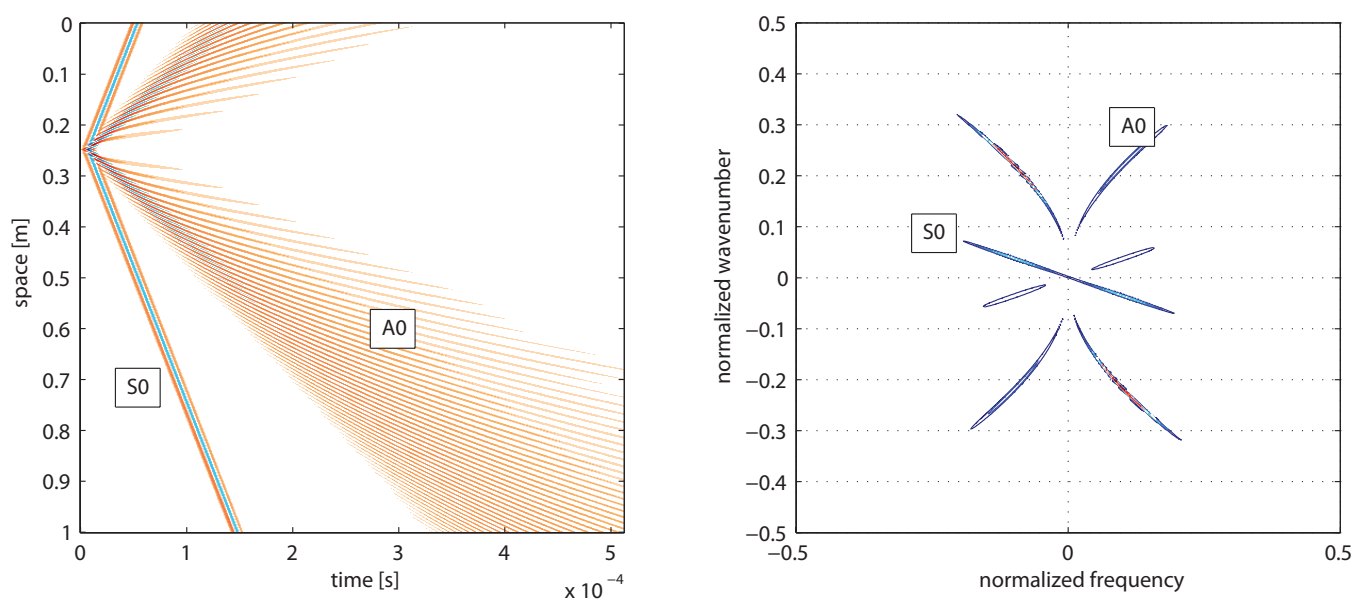

Fig. 2: Simulated propagation of Lamb waves in an aluminum plate. An acoustic source is placed at $x=0.25$. Two wave modes $\left(A_{0}\right.$ and $\left.S_{0}\right)$ are excited. Because of dispersion, the number of non-negligible samples is quite high in the space-time domain. In the Fourier domain, the signal is confined on the $k(\omega)$ curves, and the representation is consequently much more sparse.

representation, since the information is compressed in few non-negligible Fourier coefficients along the dispersion curves of two wave modes $\left(A_{0}\right.$ and $\left.S_{0}\right)$.

The sparsifying properties of the Fourier transform can be shown also by looking at Fig. 3. In this figure, such operator is applied to the spatial wavefield captured on a given instant in a simulated setup similar to the previous one (although just the A0 wave is captured in the considered snapshot). It is worth noticing how the transform produces a very sparse representation in the wavenumber domain.

The representation offered by the Fourier transform has been fruitfully used ([24]) to separate the waves scattered by defects from the incident ones. Fourier bases are consequently good candidates for the construction of the sparsifying dictionary in the CS recovery procedure. It is also worth noticing that the inverse Fourier transforms operator is maximally incoherent with the sampling matrix, because $\mu$ attains its minimum at $\mu=1$ for the Fourier-Dirac pair.

\section{B. Curvelets domain}

The curvelet transform (CT) [25] provides a compact architecture for sparse representation of images with singularities along curves. For a given 2D function, the transform performs a decomposition in a frame of "needle-shaped" waveforms (named curvelets) that are localized not only in position (the spatial 


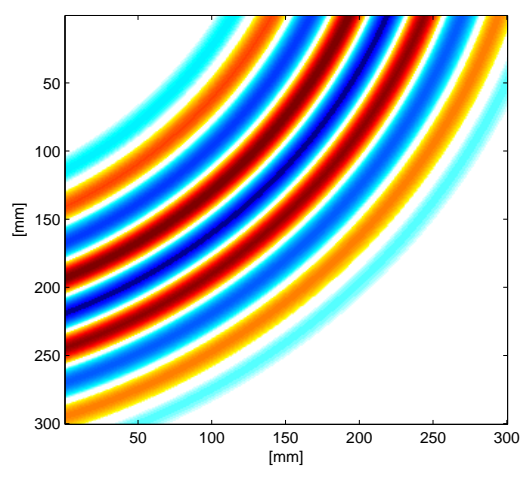

(a)

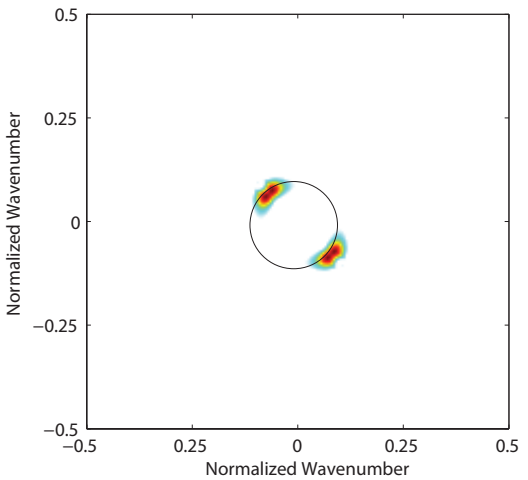

(b)

Fig. 3: Simulated propagation of Lamb waves in an aluminum plate. A snapshot of the wavefield related to a $300 \times 300 \mathrm{~mm}^{2}$ area is depicted. (a). The Fourier transform produces a very sparse representation in the wavenumber domain. (b)

domain) and scale (the wavenumber domain), but also in orientation. The curvelets construction is based on a tiling of the wavenumber domain performed by a couple of strictly bandpass classes of functions.

Let $\xi=\left(\xi_{1}, \xi_{2}\right)$ be the wavenumbers along 2D spatial axes. Curvelet functions are indexed by three parameters: a scale $\nu$, a direction $l$ and a position index $x=\left(x_{1}, x_{2}\right)$ which specifies the translation $b_{x}^{\nu, l}$ of the curvelet waveform. The product of concentric square functions $\tilde{W}_{\nu}(\xi)$ and sheared angle functions $V_{\nu}(\xi)$ defines Cartesian windows in the wavenumber domain:

$$
\tilde{U}_{\nu, l}(\xi)=\tilde{W}_{\nu}(\xi) V_{\nu}\left(S_{\theta_{l}}, \xi\right)
$$

where $S_{\theta_{l}}$ is the shear matrix

$$
S_{\theta_{l}}=\left(\begin{array}{cc}
1 & 0 \\
-\tan \theta_{l} & 1
\end{array}\right),
$$

and angles $\theta_{l}$ are chosen to define a set of equispaced slopes $\tan \theta_{l}=l \cdot 2^{\lfloor\nu / 2\rfloor}$, with $l=-2^{\lfloor\nu / 2\rfloor}, \ldots, 2^{\lfloor\nu / 2\rfloor}-$ 1. The set of windowing functions $\tilde{W}_{\nu}$ provides a decomposition of the wavenumber plane in Cartesian coronae, each divided by sheared functions $V_{\nu}$ of $N_{\nu}=4 \cdot 2^{\lceil\nu / 2\rceil}$ trapezoids. According to Fig. 4a, the number of shears doubles in each second square. On the other hand, in the space domain the wavenumber localization of $\tilde{U}_{\nu, l}$ implies the rapid decay away from a $2^{-\nu}$ by $2^{-\nu / 2}$ rectangle with major axis orthogonal to the direction $\theta_{l}$ (see Fig. 5a), so that the support's length and width obey the parabolic scaling relation

$$
\text { length } \approx 2^{-\nu / 2}, \quad \text { width } \approx 2^{-\nu} \Rightarrow \text { width } \approx \text { length }^{2},
$$


An example of curvelet atom for $\nu=, l=$ and $x=$ is provided in Fig. 5a.

In three dimensions ( 2 spatial and 1 temporal dimensions), the theory is essentially the same, except that curvelets are now 3D basis functions of side-length $2^{-\nu / 2}$ in two directions and thickness $2^{-\nu}$ in the orthonormal direction, so that the parabolic scaling relation still holds, and $\xi=\left(\xi_{1}, \xi_{2}, \xi_{3}\right)$ is now related to wavenumbers and frequency. 3D curvelets provide valuable sparse representations of 3D objects with singularities along smooth surfaces.

For a given (either 3- or 2D) signal $s$ whose Fourier transform is $S(\xi)$, the curvelet coefficients can be computed as

$$
c(\nu, l, x)=\int S(\xi) \tilde{U}_{\nu, l}(\xi) e^{i\left\langle b_{x}^{\nu, l}, \xi\right\rangle} d \xi .
$$

Therefore a practical implementation performs three steps: 1) data are transformed into the frequency domain by forward fast Fourier transform (FFT), 2) transformed data are multiplied by the set of window functions $\tilde{U}_{\nu, l}$ and 3 ) curvelet coefficients are obtained from windowed data by inverse FFT. The computational complexity is $O\left(N^{2} \log _{2} N\right)$. Further details about discrete curvelet transform implementation can be found in [25] and [26], while the code (Curvelab package) which computes the curvelet transform employed in this study is available at [27].

It has been proved that the curvelet operator is the one that produces the sparsest representation of wave propagation phenomena [28], and such property potentially allows to reduce the number of sample locations, following (3). However, Curvelets and discrete Dirac delta are less incoherent with respect to the Fourier-Dirac pair, and this may adversely affect the recovery (see [29]).

\section{Wave Atoms domain}

Wave atoms [30] represent another tool for multiscale analysis, allowing to expand a multidimensional function in a linear combination of localized, directional waveforms. Similarly to curvelets, wave atoms provide a tiling of the frequency/wavenumber plane (see Fig. 4(b)) which has been proven to sparsely represent oscillatory patterns [30].

In the space domain, wave atoms are smoothed oscillating functions whose support is approximately a square of size $2^{-\nu}$ by $2^{-\nu}$ at scale $\nu$, with oscillation of wavelength $\sim 2^{-2 \nu}$ transverse to the ridge, while in frequency domain each tile is a $2^{\nu}$ by $2^{\nu}$ square, at a distance $\sim 2^{2 \nu}$ from the origin, as showed in Fig. 4(b).

Note that the subscript $\nu$ indexes the different "dyadic coronae", whereas direction and position are indexed by $l$ and $x$, respectively. Fig. 5(b) exhibits a wave atom for $\nu=, l=$ and $x=$, which shows a more isotropic aspect ratio w.r.t. curvelet in Fig. 5(a). 
All the transformations considered in this section (including curvelets and wave atoms) make a central use of discrete Fourier transform algorithms such as the Fast Fourier Transform (FFT). Due to its implicit periodicity, FFT may cause reconstruction artifacts across boundaries. A way of avoiding this unwanted outcome is to work on the discrete cosine transform of the original domain, in place of the Fourier transform, with no penalty on redundancy or computational complexity. The complete mathematical treatment can be found in [31], while the code used in this study is available at [32].

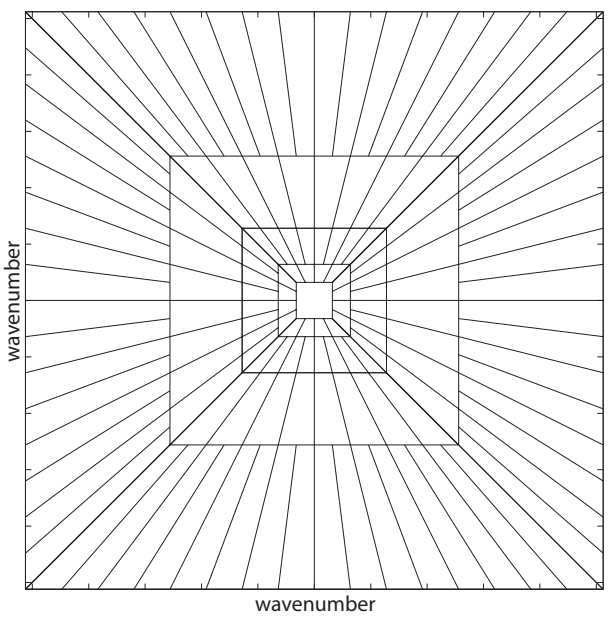

(a)

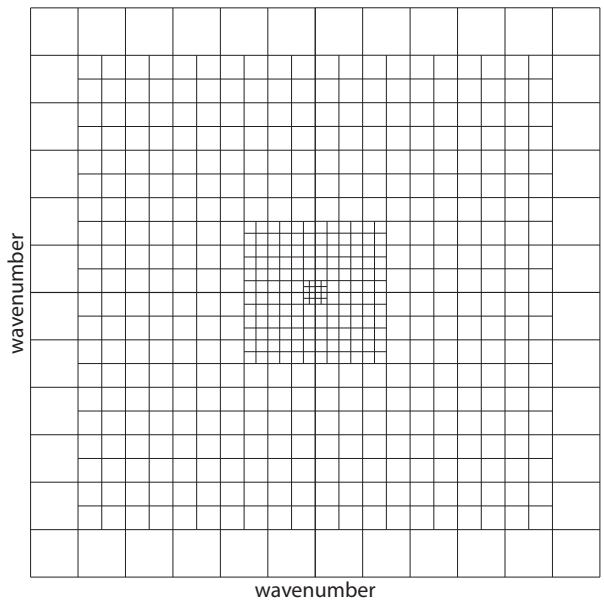

(b)

Fig. 4: (a) Curvelet dyadic decomposition of the wavenumber plane; (b) wave atom tiling of the wave number plane.

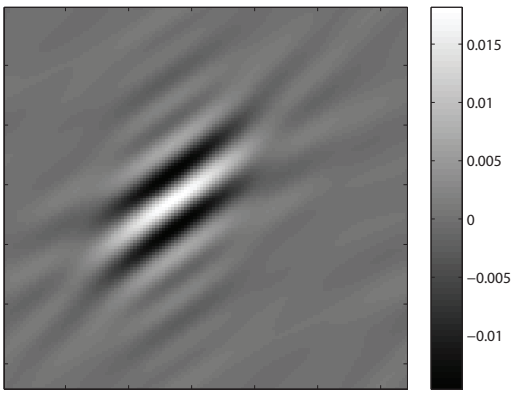

(a)

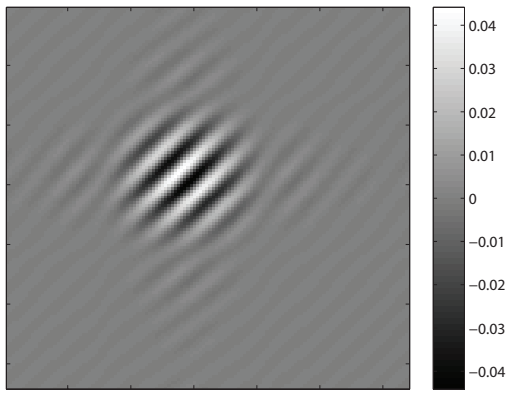

(b)

Fig. 5: Examples of basis functions: (a) curvelet atom; (b) wave atom 


\section{EXPERIMENTAL RESULTS AND DISCUSSION}

The results of the CS reconstruction of a wavefield signal acquired on two distinct setups were evaluated in order to assess the proposed approach. The first setup is constituted by a $1.5 \mathrm{~mm}$ thick 6061 aluminum plate $(638 \mathrm{~mm} \times 558 \mathrm{~mm}$ wide). The presence of a defect was emulated by a $12 \mathrm{~mm}$ diameter cylindrical mass bonded on the surface of the plate. A $10 \mathrm{~mm}$ diameter PZT transducer was used to excite the guided field in the structure, while the plate response was recorded through a Polytec PSV400M2 SLDV over a rectangular area of $150 \mathrm{~mm} \times 155 \mathrm{~mm}$, in a grid of $141 \times 151$ equispaced points.

The second setup is constituted by a glass fibre reinforced polymer (GFRP) plate (4 unidirectional glass fibre layers oriented along $\mathrm{x}$ axis, thickness equal to $3.2 \mathrm{~mm}$ ). Three $0.5 \mathrm{~mm}$ wide and $70 \%$ of the specimen thickness deep notch cuts were machined by a sharp tool. The excitation signal was generated by an arbitrary waveform generator, amplified to $400 \mathrm{Vpp}$ by a dedicated PZT amplifier. The excitation signal was applied to a round piezoelectric transducer $(10 \mathrm{~mm}$ diameter). Out of plane displacements were registered by a SLDV on dense grid of $(385 \times 389)$ equally spaced points.

The CS procedure was implemented in Matlab using the SPGL1 toolbox [33] which iteratively solves the sparsity-promoting problem in Eq. (5), with the support of the suite Sparco [34]. The outcomes of the procedure were compared in terms of both computational time and Signal to Noise Ratio (SNR), defined as

$$
20 \cdot \log _{10}\left(\frac{\left\|u\left(x_{1}, x_{2}, t\right)\right\|}{\left\|u_{r}\left(x_{1}, x_{2}, t\right)-u\left(x_{1}, x_{2}, t\right)\right\|}\right),
$$

where $u$ is the signal acquired on the dense grid, and $u_{r}$ is the recovered wavefield signal, by varying the number of iterations of the SPGL1 algorithm (detailed in Appendix B), and the sparsifying basis.

To qualitatively judge the performance of the presented approach, the recovery of two snapshots related to the different setups are illustrated in Fig. 6 and 7. In Fig. 6(a), the signal acquired on the aluminum plate at a given time instant is shown along with the ones recovered with different dictionaries (Fig. 6(c)-(f)). The recovery was performed starting from less than $34 \%$ measurements with respect to the original grid (the JS mask is illustrated in Fig. 6(b) with black square dots). It is worth noticing the substantial agreement of all the recovered signals to the acquired one, which proves the effectiveness of the procedure. Furthermore, the presented results are much more accurate than the ones achieved by simply interpolating the sub-sampled signal, as can be seen by looking at the linear interpolation results depicted in Fig. 6(c). Similarly, the recovery of the wavefield acquired on the composite panel is very effective, as can be seen in Fig. 7.

In Fig. 8, the results of the implemented procedure applied to the first setup are depicted in terms of 


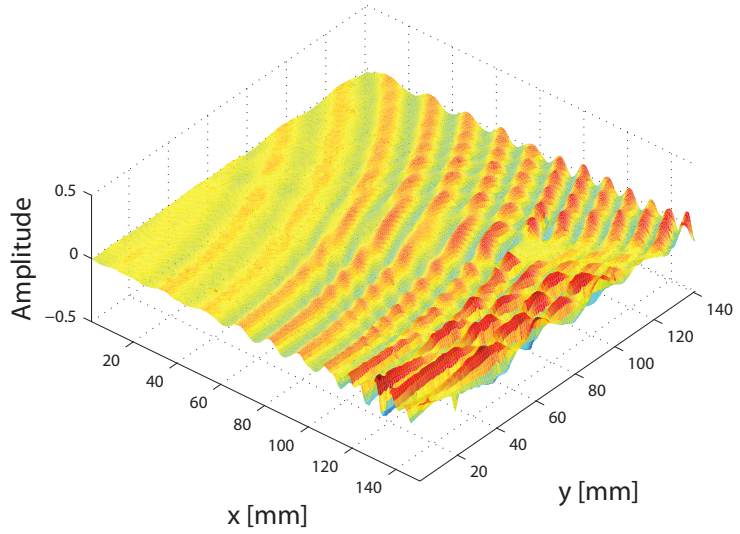

(a)

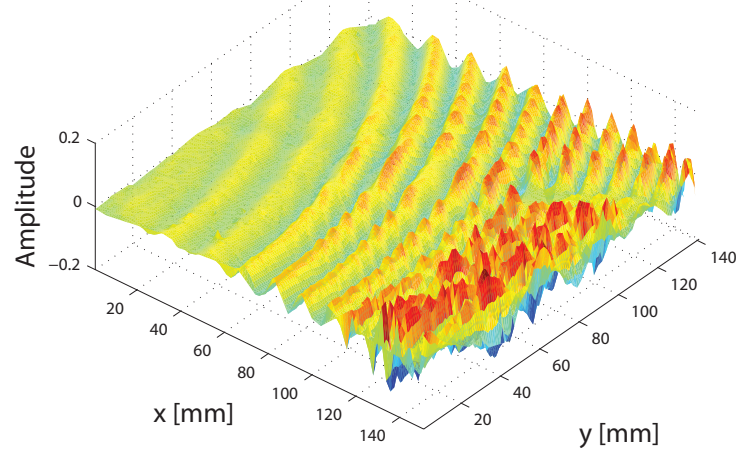

(c)

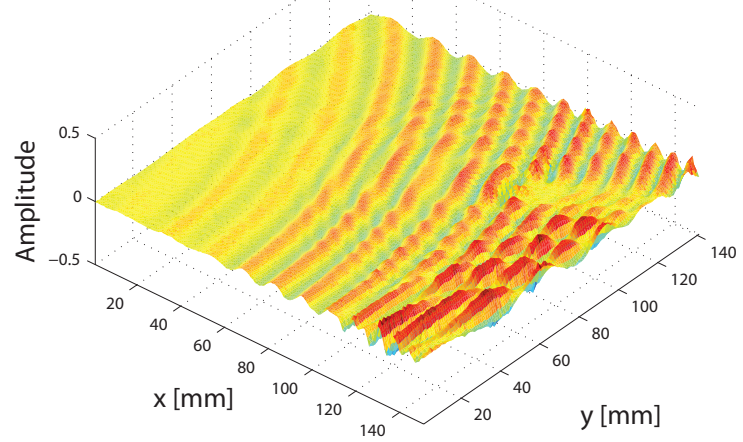

(e)

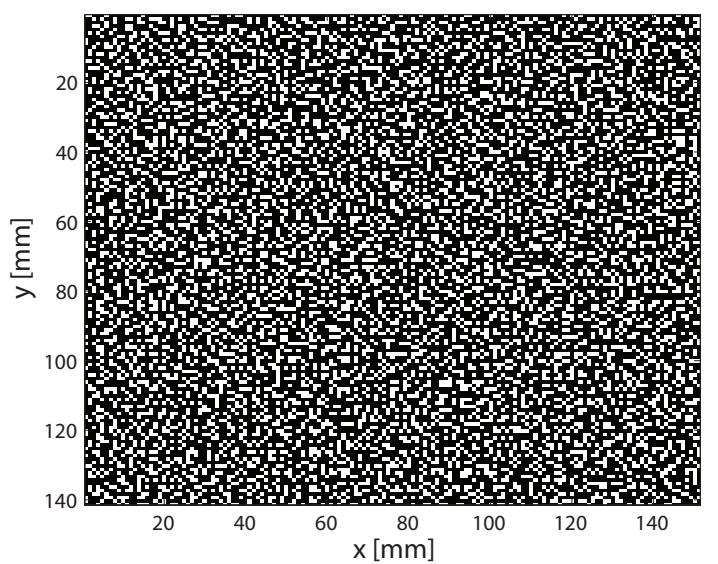

(b)

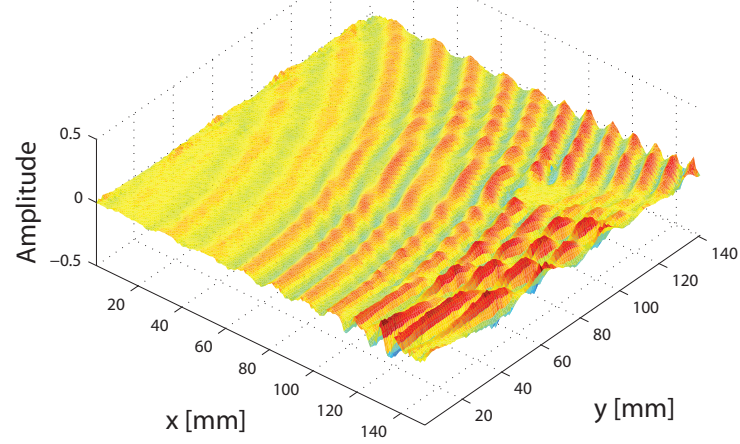

(d)

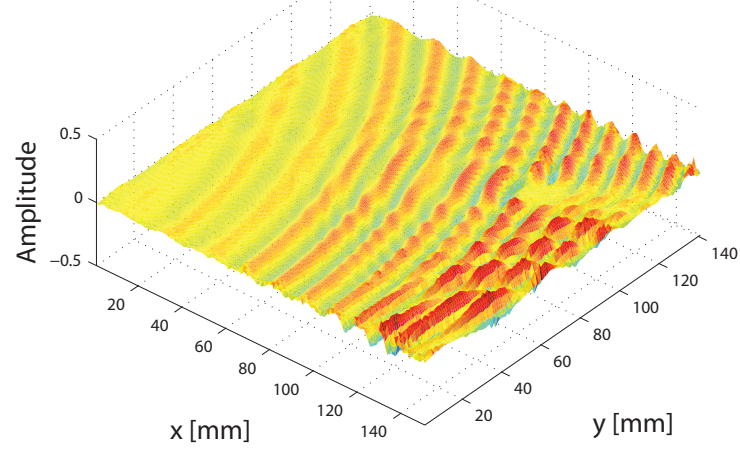

(f)

Fig. 6: (a) Original full wavefield on aluminum plate at a given time instant, (b) JS mask used to undersample the acquisitions, (c) linear interpolation, (d) Full wavefield recovered with FFT 3D, (e) Wave Atom, (f) 2D Curvelet domain by less than $34 \%$ measurements respect to the original sampling
February 10,2015 grid. 


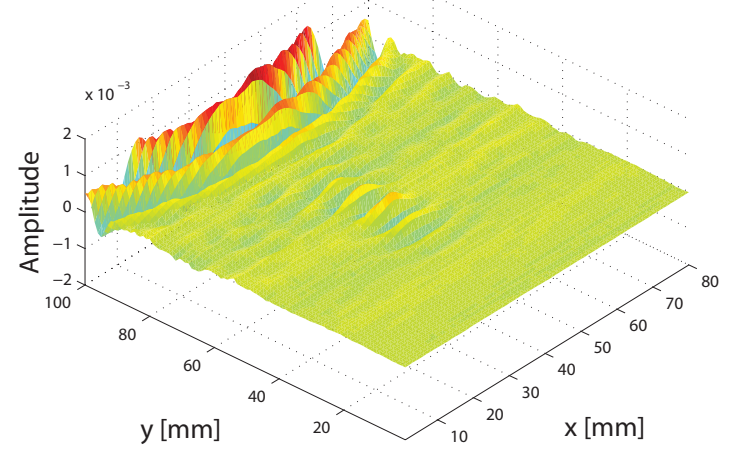

(a)

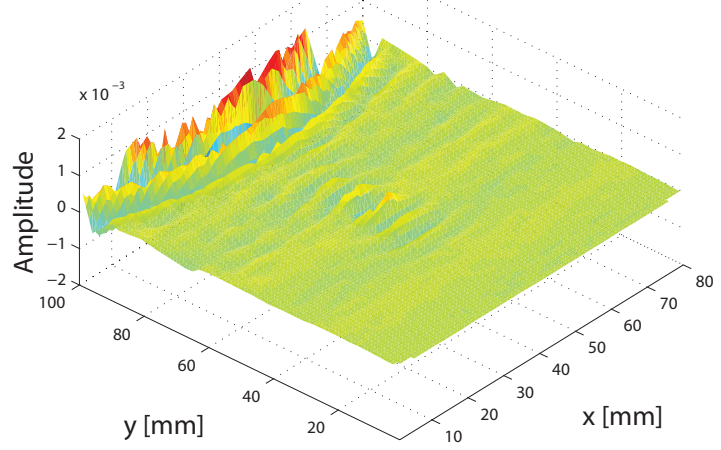

(c)

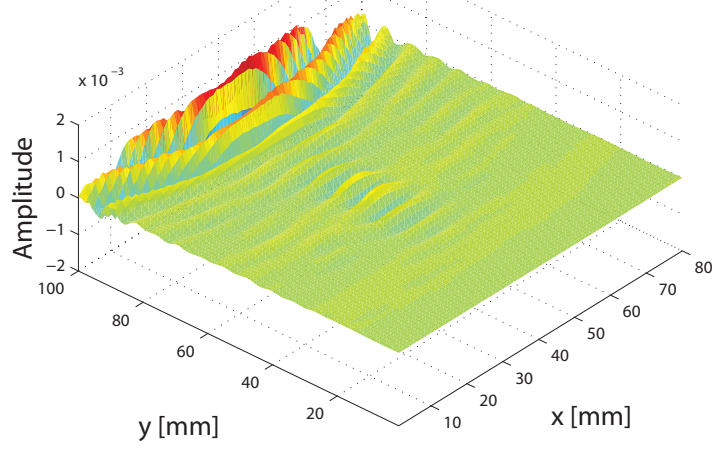

(e)

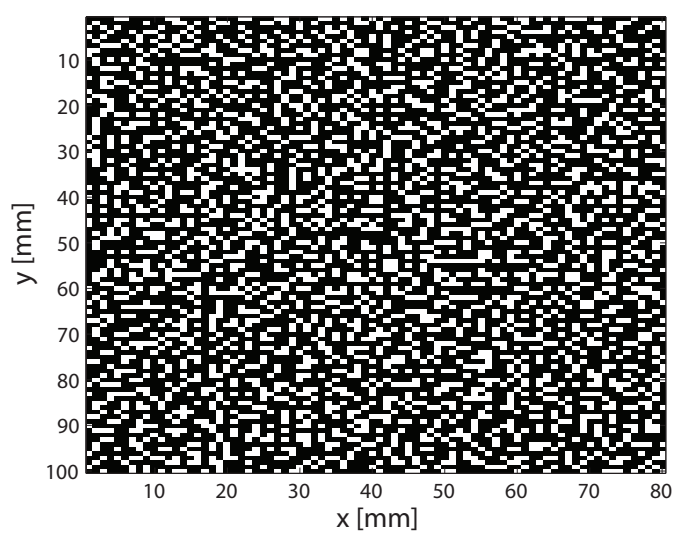

(b)

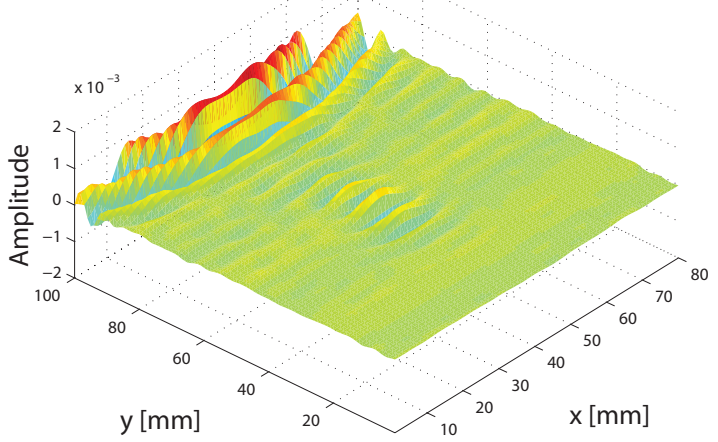

(d)

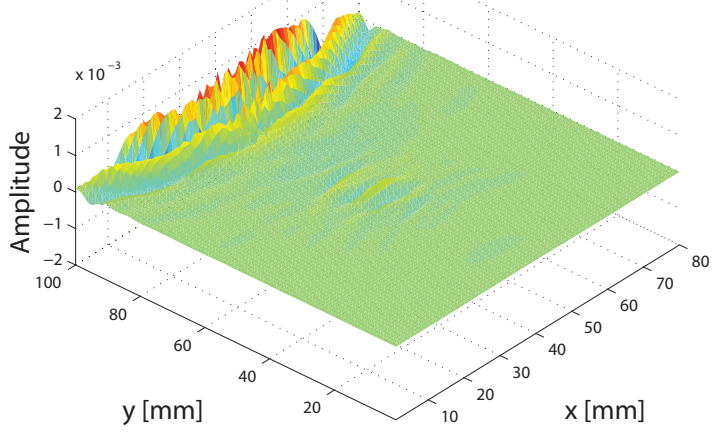

(f)

Fig. 7: (a) Original full wavefield on composite plate at a given time instant, (b) JS mask used to undersample the acquisitions, (c) linear interpolation, (d) Full wavefield recovered with FFT 3D, (e) Wave Atom, (f) 2D Curvelet domain by less than $34 \%$ measurements respect to the original sampling Februlary 10, 2015

DRAFT grid. 
As can be observed in Table I, the number of SPGL1 iterations is not directly proportional to the computational time, so that the percentage of retained scanpoints just partially influences the computational cost of the reconstruction procedure.

In Table II the results of the reconstruction of the second setup wavefield signal in the 3D Fourier basis with 30 iterations by means of Random, Jittered and FPS distributions are shown. For each sampling strategy, 20 realizations were considered. The minimum and maximum values of the achieved SNRs are reported, retaining the $15 \%$ and $33 \%$ of the original grid scanpoints. Even though the Farthest Point 


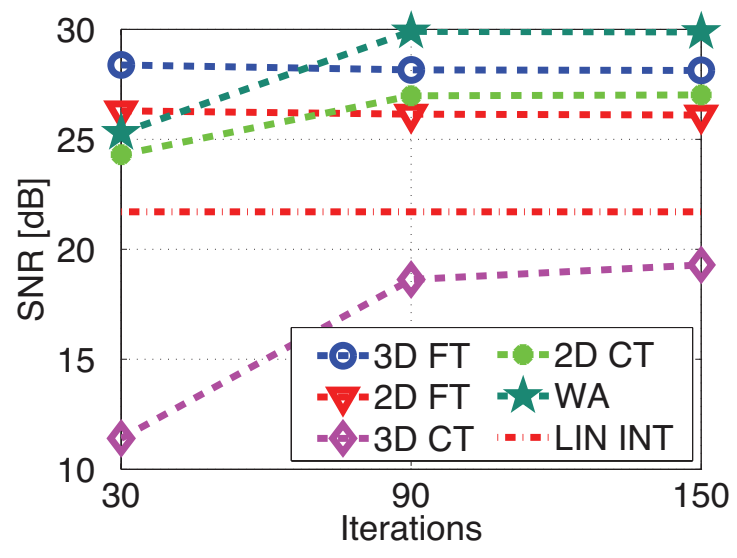

(a)

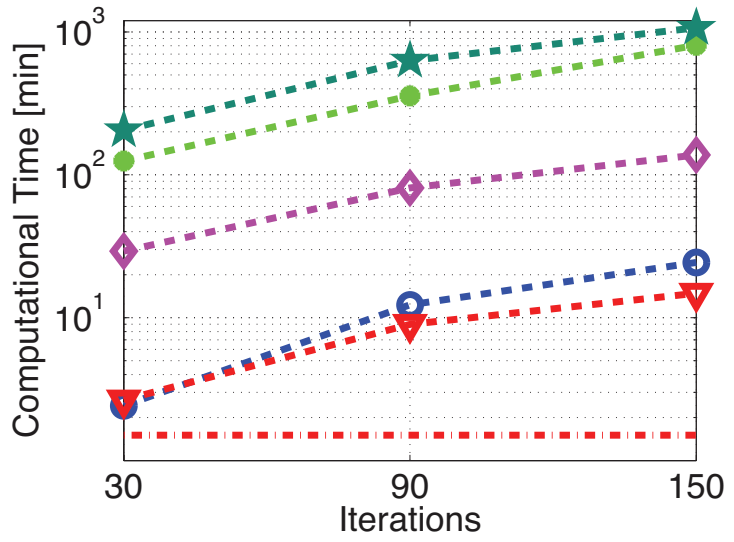

(b)

Fig. 8: (a) SNR performances achieved, and (b) computational time needed to recover the full wavefield with different sparsifying basis as a function of the number of iterations of the SPGL1 algorithm. The dot-dashed lines indicate the performances achieved by the simple linear interpolation.

TABLE I: Computational Time of the CS recovery obtained from: a) full wavefield signal random subsampled in 33\% locations respect to the original grid distributed with 2D Jittered approach (30 iteration for the SPGL1 algorithm); b) considering 90\% of the grid points (15 iteration for the SPGL1 algorithm).

\begin{tabular}{lcc} 
Dictionary & Computational Time (a) [min] & Computational Time (b) [min] \\
\hline \hline 3D FFT & 12.31 & 9.03 \\
\hline 2D FFT & 8.99 & 6.57 \\
\hline 3D CT & 81.00 & 71.27 \\
\hline
\end{tabular}


TABLE II: Reconstruction SNRs in the Fourier domain after 30 iterations of the CS problem solver for different sampling-point distribution strategies (15\% or 33\% samples preserved, 20 grids generated for each strategy).

\begin{tabular}{|l|c|c|c|c|}
\hline \multirow{2}{*}{$\begin{array}{l}\text { Sampling } \\
\text { scheme }\end{array}$} & \multicolumn{2}{|c|}{ SNR $[\mathrm{dB}](15 \%)$} & \multicolumn{2}{c|}{ SNR [dB] (33\%) } \\
\cline { 2 - 5 } & $\min$ & $\max$ & $\min$ & $\max$ \\
\hline Random & 15.94 & 17.37 & 27.72 & 29.46 \\
\hline Jittered & $\mathbf{1 6 . 6 3}$ & $\mathbf{1 7 . 5 5}$ & $\mathbf{2 9 . 4 3}$ & $\mathbf{3 0 . 7 4}$ \\
\hline FPS & 14.46 & 15.49 & 24.83 & 26.23 \\
\hline
\end{tabular}

an alternative weighted-metric over the spatial domain, therefore providing a valuable adaptive sampling scheme (see Sec. III, and [17]).

\section{Conclusions}

In this paper, a CS framework for acoustic wavefield acquisitions was presented. The proposed framework aims at minimizing the number of scan-point locations over the surface of the inspected structure. Both sampling-point distribution strategies and sparsity-promoting dictionaries were investigated in order to produce the best recovery for a sub-sampled wavefield signal obtained with a scanning laser Doppler vibrometer.

The decomposition of the signal in the Fourier domain turned out to be the more effective solution, leading to a very fast and accurate recovery starting from less than 34\% of measurements with respect to the original sampling grid. This result can be ascribed (i) to the fast algorithms which compute the Fourier transform, (ii) to the sparse representation of Lamb wave signals offered by the Fourier domain, as well as (iii) to the high incoherence of Fourier exponentials with the examined sampling schemes, and demonstrates the great potential of the CS approach.

Among the future developments, an adaptive sampling-point distribution strategy could be developed taking advantage of the progressiveness of the FPS scheme. It is possible indeed to introduce case-specific metrics in order to place each new sampling-point in the position that minimize the local recovery error, further improving the tradeoff between the number of scan-points and accuracy in the reconstructions.

\section{APPENDiX A: JiTTERED SUbSAMPLING SCHEME}

Let us assume that the full resolution grid is a cartesian grid of scanpoints in which each vertex has coordinates $(i * \Delta, j * \Delta)$, with $i \in[0,1, \ldots, I], j \in[0,1, \ldots, J]$ and $\Delta$ being the spatial spacing (set 
according to the Nyquist sampling theorem). If $\gamma$ is the downsampling factor, the coordinates of the Jittered subsampled grid are given by:

$$
\begin{aligned}
& x_{1}=i_{s} \cdot \frac{\Delta}{\sqrt{\gamma}}+\epsilon_{i}, i_{s} \in[0,1, \ldots, I \cdot \sqrt{\gamma}] \\
& x_{2}=j_{s} \cdot \frac{\Delta}{\sqrt{\gamma}}+\epsilon_{j}, j_{s} \in[0,1, \ldots, J \cdot \sqrt{\gamma}]
\end{aligned}
$$

where the random variables $\epsilon_{i}$ and $\epsilon_{j}$ are independently and identically distributed (i.i.d.) according to a uniform distribution on the interval $[-\Delta / \sqrt{\gamma}, \Delta / \sqrt{\gamma}]$.

\section{APPENDIX B: SPGL1 ALGORITHM}

The Spectral Projected Gradient for $\ell_{1}$ minimization (SPGL1) algorithm computes the solution of 5 by solving the sequence of the following subproblems:

$$
\alpha^{\tau_{i}}=\arg \min _{\tilde{\alpha} \in R^{n}}\|\Phi \Psi \tilde{\alpha}-y\|_{\ell_{2}} \quad \text { subject to } \quad\|\tilde{\alpha}\|_{\ell_{1}} \leq \tau_{i}
$$

The algorithm starts by defining the first tentative solution $\alpha^{0}$ and the initial value for the parameter $\tau_{0}=\left\|\alpha^{0}\right\|_{\ell_{1}}$. Then the updating of $\tau$ is performed by traversing the Pareto curve described by the function $\eta(\tau)=\left\|\mathbf{\Phi} \boldsymbol{\Psi} \alpha^{\tau_{i}}-y\right\|_{\ell_{2}}$ :

$$
\tau_{i+1}=\tau_{i}+\frac{\eta\left(\tau_{i}\right)-\epsilon}{\eta^{\prime}\left(\tau_{i}\right)}
$$

The iterations are stopped when $\left\|\Phi \Psi \alpha^{\tau_{i}}-y\right\|_{\ell_{2}}=\epsilon$ or some alternative stopping conditions (e.g. the maximum number of iterations) are met.

\section{ACKNOWLEDGEMENTS}

The authors gratefully acknowledge Prof. Ruzzene (Georgia Institute of Technology), and Prof. Ostachowicz (Polish Academy of Sciences) for the provided SLDV acquisitions. The research leading to these results has been partially supported from the SARISTU project (Grant Agreement no. 284562) and from the Harmonia project (National Science Centre Poland funds, decision number DEC-2012/06/M/ST8/00414). 


\section{REFERENCES}

[1] A. Raghavan and C. E. S. Cesnik, "Review of guided-wave structural health monitoring," The Shock and Vibration Digest, vol. 39, no. 2, pp. 91-114, March 2007.

[2] J. L. Rose, “A baseline and vision of ultrasonic guided wave inspection potential," Journal of Pressure Vessel Technology, vol. 124, pp. 273-282, August 2002.

[3] H. Sohn, D. Dutta, J. Y. Yang, M. D. Simio, S. Olson, and E. Swenson, "Automated detection of delamination and disbond from wavefield images obtained using a scanning laser vibrometer," Smart Materials and Structures, vol. 20 , March 2011.

[4] P. Sriram, S. Hanagud, and J. I. Craig, "Scanning laser doppler techniques for vibration testing," Experimental Techniques, vol. 16, no. 6, pp. 21-26, November 1992.

[5] W. Staszewski, B. Lee, L. Mallet, and F. Scarpa, "Structural health monitoring using scanning vibrometry: I. lamb wave sensing," Smart Materials and Structures, vol. 13, no. 2, pp. 251-260, February 2004.

[6] E. Candès and M. Wakin, "An introduction to compressive sampling," IEEE Signal Processing Magazine, vol. 25, no. 2, pp. 21-30, March 2008.

[7] H. Liebgott, R. Prost, and D. Friboulet, "Pre-beamformed RF signal reconstruction in medical ultrasound using compressive sensing," Ultrasonics, vol. 53, no. 2, pp. 525-533, 2013.

[8] M. F. Schiffner and G. Schmitz, "Fast pulse-echo ultrasound imaging employing compressive sensing," in Proc. IEEE Int. Ultrasonics Symposium (IUS), 2011, pp. 688-691.

[9] C. Quinsac, A. Basarab, and D. Kouamé, "Frequency domain compressive sampling for ultrasound imaging," Advances in Acoustics and Vibration, vol. 2012, 2012.

[10] N. Wagner, Y. C. Eldar, and Z. Friedman, “Compressed beamforming in ultrasound imaging," Signal Processing, IEEE Transactions on, vol. 60, no. 9, pp. 4643-4657, 2012.

[11] W. Tang, J. Ma, and F. J. Herrmann, “Optimized compressed sensing for curvelet-based seismic data reconstruction," preprint, vol. 280, 2009.

[12] F. J. Herrmann, M. P. Friedlander, and O. Yilmaz, "Fighting the curse of dimensionality: compressive sensing in exploration seismology," Signal Processing Magazine, IEEE, vol. 29, no. 3, pp. 88-100, 2012.

[13] A. Perelli, T. Di Ianni, A. Marzani, L. De Marchi, and G. Masetti, "Model-based compressive sensing for damage localization in lamb wave inspection," Ultrasonics, Ferroelectrics and Frequency Control, IEEE Transactions on, vol. 60, no. $10,2013$.

[14] J. Harley and J. Moura, "Sparse recovery of the multimodal and dispersive characteristics of Lamb waves," Journal of the Acoustical Society of America, vol. in press.

[15] D. Mascarenas, S. Chong, G. Park, J. Lee, and C. Farrar, "Application of compressed sensing to 2-d ultrasonic propagation imaging system data," in Proc. 6th European Workshop on Structural Health Monitoring, 2012, pp. 1-8.

[16] R. Rubinstein, A. M. Bruckstein, and M. Elad, "Dictionaries for sparse representation modeling," Proceedings of the IEEE, vol. 98, no. 6, pp. 1045-1057, 2010.

[17] Y. Eldar, M. Lindenbaum, M. Porat, and Y. Zeevi, "The farthest point strategy for progressive image sampling," IEEE Transactions on Image Processing, vol. 6, no. 9, pp. 1305-1315, September 1997.

[18] E. Candès, J. Romberg, and T. Tao, "Robust uncertainty principles: Exact signal reconstruction from highly incomplete frequency information," IEEE Transactions on Information Theory, vol. 52, no. 2, pp. 489-509, February 2006.

[19] E. Candes and J. Romberg, "Sparsity and incoherence in compressive sampling," Inverse problems, vol. 23, no. 3, p. 969, 2007. 
[20] G. Hennefent and F. J. Herrmann, "Simply denoise: wavefield reconstruction via jittered under-sampling," Geophysics, vol. 73, no. 3, pp. 19-28, May 2008.

[21] R. L. Cook, "Stochastic sampling in computer graphics," ACM Transactions on Graphics, vol. 5, no. 1, pp. 51-72, January 1986.

[22] A. Okabe, B. Boots, and K. Sugihara, Spatial Tessellations - Concepts and Applications of Voronoi Diagrams. John Wiley \& Sons, Chicester, 2000.

[23] A. Marzani and S. Salamone, "Numerical prediction and experimental verification of temperature effect on plate waves generated and received by piezoceramic sensors," Mechanical Systems and Signal Processing, vol. 30, no. 0, pp. 204 217, 2012.

[24] M. Ruzzene, "Frequency-wavenumber domain filtering for improved damage visualization," Smart Materials and Structures, vol. 16, pp. 2116-2129, October 2007.

[25] J. Ma and G. Plonka, "The curvelet transform," IEEE Signal Processing Magazine, vol. 27, no. 2, pp. 118-133, March 2010.

[26] E. Candès, L. Demanet, D. Donoho, and L. Ying, "Fast discrete curvelet transforms," Applied and Computational Mathematics, California Institute of Technology, Tech. Rep., 2005.

[27] [Online]. Available: http://www.curvelet.org

[28] E. J. Candes and L. Demanet, "The curvelet representation of wave propagators is optimally sparse," Communications on Pure and Applied Mathematics, vol. 58, no. 11, pp. 1472-1528, 2005.

[29] F. J. Herrmann and G. Hennenfent, "Non-parametric seismic data recovery with curvelet frames," Geophysical Journal International, vol. 173, no. 1, pp. 233-248, 2008.

[30] L. Demanet, "Curvelets, wave atoms and wave equations," Ph.D. dissertation, California Institute of Technology, 2006.

[31] L. Demanet and L. Ying, "Curvelets and wave atoms for mirror-extended images," Proc. SPIE, Wavelets XII, vol. 6701, no. 67010J, September 2007.

[32] [Online]. Available: http://www.waveatom.org

[33] E. van den Berg and M. P. Friedlander, "Probing the pareto frontier for basis pursuit solutions," SIAM J. on Scientific Computing, vol. 31, no. 2, pp. 890-912, November 2008.

[34] E. van den Berg, M. P. Friedlander, F. J. Herrmann, R. Saab, and O. Yilmaz, "Sparco: A testing framework for sparse reconstruction,” Department of Computer Science, University of British Columbia, Tech. Rep., 2007.

[35] L. D. Marchi, E. Baravelli, M. Ruzzene, N. Speciale, and G. Masetti, "Guided wave expansion in warped curvelet frames," IEEE Transactions on Ultrasonics, Ferroelectrics and Frequency Control, vol. 59, no. 5, pp. 949-957, May 2012.

[36] R. Shahidi, G. Tang, J. Ma, and F. J. Herrmann, "Application of randomized sampling schemes to curvelet-based sparsitypromoting seismic data recovery," Geophysical Prospecting, vol. 61, no. 5, pp. 973-997, September 2013.

Tommaso Di Ianni was born in Torremaggiore, Foggia, Italy, in 1988. He received the Master of Science degree in Electronic Febrineering in 2014 at the University of Bologna with a thesis on Compressive Sensing based Damage Detection. Actually he is pursuing the Ph.D.degree at the Technical University of Denmark. 
Luca De Marchi received the Dr.Eng. and Ph.D. Degrees in Electronic Engineering respectively in 2002 and 2006 from the University of Bologna. At the end of 2002, he joined the Department of Electronics, Computer Sciences and Systems (DEI) at the University of Bologna. Currently, he is also with the Advanced Research Center for Electronic Systems (ARCES). His current research interests are in ultrasonic signal analysis for structural health monitoring applications.

Alessandro Perelli was born in Senigallia, Ancona, Italy, in 1985. He received the Master of Science degree in Electronic Engineering in October 2010 and his Bachelor of Science in Electronic Engineering in 2007, both at the University Polytechnic of Marche, Ancona (Italy). Since September 2012 he has been a visiting research scholar at the Ultrasound Group of University of Leeds. In 2014 he received the Ph.D. degree in Electronic Engineering at the Department of Electronics, Computer Science and Systems DEI - University of Bologna. Since 2014 is Research Associate at the University of Edinburgh.

Alessandro Marzani received the B.S. in civil eng. from the Univ. of Bologna, Italy, in 2001; the M.S. in structural eng. from the Univ. of California, San Diego, USA, in 2004; and the Ph.D. in eng. of materials and structures from the Univ. of Calabria, Cosenza, Italy, in 2005. Since 2014, he is an Associate Prof. of Structural Mechanics at the Department of Civil, Chemical, Environmental and Materials Engineering (DICAM), Univ. of Bologna. His research interests include guided wavebased nondestructive and monitoring techniques of materials and structures, signal processing strategies, and numerical modeling. 